\title{
Cryptococcus gattii Meningoencephalitis in an Immunocompetent Person 13 Months after Exposure
}

\author{
A. Georgi, M. Schneemann, K. Tintelnot, R.C. Calligaris-Maibach, \\ S. Meyer, R. Weber, P.P. Bosshard
}

\begin{abstract}
A 53-year old immunocompetent Swiss female is described who developed severe meningoencephalitis due to infection with Cryptococcus gattii 13 months following exposure on Vancouver Island, Canada. Diagnosis was based on cerebrospinal fluid (CSF) examination, i.e., positive India-ink staining, positive latex particle agglutination, and positive culture. Species identification was performed by growth on L-canavanine-glycine-bromthymol blue medium and by sequencing of the intergenic and internal transcribed spacer regions of the rRNA genes. After initial therapy with fluconazole by which the patient did not improve, therapy was changed to amphotericin B and flucytosine and later to high-dose fluconazole and amphotericin B. Despite long-term treatment and external drainage of the CSF, the patient's condition improved only slowly. The patient was discharged after 132 days of hospitalization.
\end{abstract}

Infection 2009; 37: 370-373

DOI 10.1007/s15010-008-8211-z

\section{Introduction}

The etiologic agent of cryptococcosis is classified into two species: Cryptococcus neoformans (serotype A, AD and D) and Cryptococcus gattii (serotype B and C; formerly C. neoformans var. gattii) [1]. Both are encapsulated environmental yeasts which, however, differ in their natural habitat and geographical distribution. In nature, $C$. neoformans has been isolated all over the world from soil contaminated with pigeon or other bird droppings. C. gattii has been isolated from decaying wood, mainly of Eucalyptus trees, with the distribution of human infections corresponding in general to the distribution of these trees, i.e., tropical and subtropical areas such as Australia, Central and South America, India, China, and limited areas in Europe [2]. However, in 1999, a C. gattii outbreak emerged on Vancouver Island, Canada [3].

C. neoformans tends to infect - although not exclusively - immunodeficient people, such as HIV-infected individuals and those with lymphoproliferative disorders or on corticosteroid treatment. Infection typically mani- fests as diffuse meningitis or meningoencephalitis. In contrast, $C$. gattii preferably infects immunocompetent hosts, with the major risk factor appearing to be environmental exposure [4]. In $C$. gattii infection, cerebral mass lesions and/or hydrocephalus, pulmonary mass lesions, increased neurological deficits, and a slower response to treatment are more common than in $C$. neoformans infection [4].

\section{Case Report}

A 53-year-old woman with no relevant prior medical history was admitted to hospital in October 2006 because of fever and persistent headache for 1 month, walking disturbances, and vision disorder. During the physical examination, she suffered a partial epileptic seizure. A CT scan of the brain was normal, but the pressure of the CSF was markedly elevated $\left(46 \mathrm{~cm} \mathrm{H}_{2} \mathrm{O}\right)$. Analyses of the CSF showed a cell count of $100 \mu \mathrm{l}^{-1}$, increased protein $(1.5 \mathrm{~g} / \mathrm{l})$, and reduced glucose. Empiric intravenous antimicrobial therapy with ceftriaxon, amoxicillin, and acyclovir was started (Figure 1). The CSF was subsequently reported to be positive for cryptococcal antigen (Meridian Diagnostics, Cincinnati, OH) (titer $>1: 2,048$; Figure 1). Staining of the CSF with India ink (or with periodic acid-Schiff reagent) showed abundant encapsulated yeast cells upon

\footnotetext{
A. Georgi, M. Schneemann

Dept. of Medicine, University Hospital Zurich, Zurich, Switzerland

K. Tintelnot

Robert Koch Institute, Berlin, Germany

R.C. Calligaris-Maibach, P.P. Bosshard

Institute of Medical Microbiology, University of Zurich, Zurich, Switzerland

S. Meyer

Dept. of Medicine, Cantonal Hospital, Glarus, Switzerland

R. Weber

Division of Infectious Diseases and Hospital Epidemiology, Department of Medicine, University Hospital Zurich, Zurich, Switzerland

P.P. Bosshard (corresponding author)

Dept. of Dermatology, University Hospital Zurich, Gloriastrasse 31,

8091 Zurich, Switzerland; Phone: (+41/44) 255-3972, Fax: -4418,

e-mail: philipp.bosshard@usz.ch
}

Received: May 26, 2008 - Revision accepted: October 16, 2008

Published online: April 23, 2009 
Figure 1. Cryptococcal antigen titer in CSF (filled square) and serum (filled diamond), antimicrobial treatment, and periods of external ventricular shunt. $\mathrm{X}$-axis: date; Y-axis: titer; CAA: ceftriaxon, amoxicillin, and acyclovir; $\mathrm{F}$ : fluconazole; AFy: amphotericin B and flucytosine; AF: amphotericin B and fluconazole.

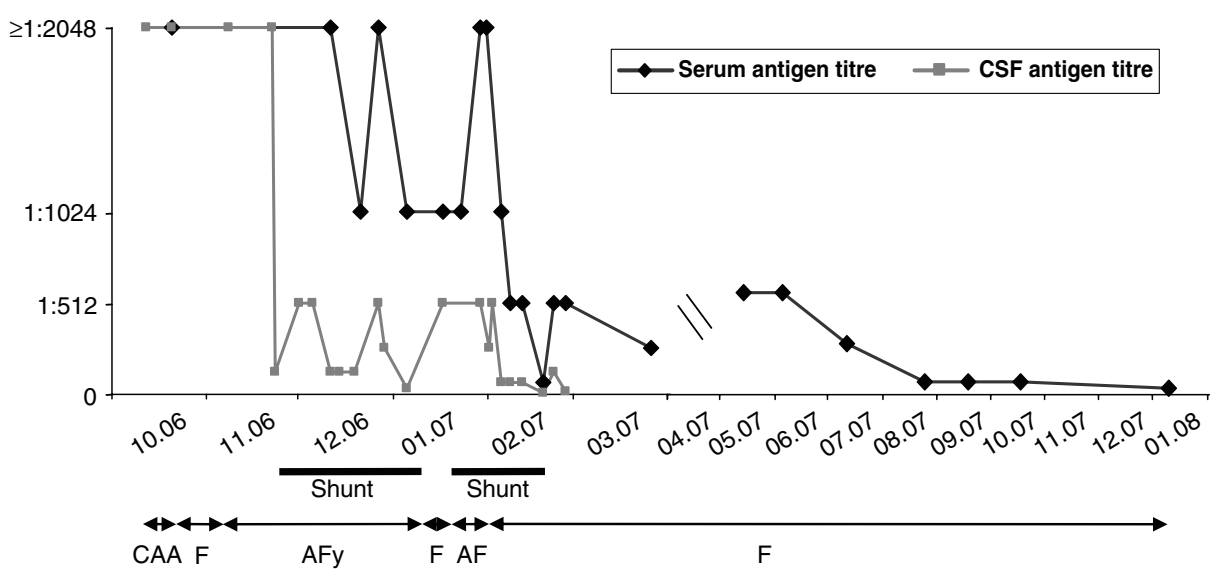

microscopic evaluation. Intravenous antibiotics were stopped and oral therapy with fluconazole (400 mg/day) was started. Fluconazole was chosen as the initial therapy because the patient appeared not to be severely ill at this time. The yeast cells of the CSF culture were biochemically identified with the Auxacolor system (Sanofi Diagnostics Pasteur, Freiburg, Germay) as Cryptococcus neoformans. Based on the ability of the cells to grow on CGB medium and on sequence analysis of the intergenic spacer 1 region (IGS1), we identified the isolate as C. gattii [5]. The IGS sequence was assigned to the IGS genotype 3 [6]. The isolate was serotyped by CryptoCheck (Iatron Laboratories, Tokyo, Japan) as serotype B, and this identification was confirmed by Fourier transform infrared (FT-IR) spectroscopy [7]. Sequence analysis of the internal transcribed spacer (ITS) regions revealed that the isolate belonged to the ITS type 4 according to Katsu et al. [8], which correlates to the genotype VGII [8, 9]. Susceptibility testing was performed using the Sensititre YeastOne plate (Trek Diagnostics Systems, East Grinstead, UK). The results were as follows (in $\mu \mathrm{g} / \mathrm{ml}$ ): amphotericin B, 0.5; flucytosine, 2.0; fluconazole, 8.0; itraconazole, 0.064, ketokonazole, 0.032; voriconazole, 0.064 .

Due to clinical deterioration of the patient, after 18 days we switched the oral regimen to amphotericin B iv $(1 \mathrm{mg} / \mathrm{kg}$ of body weight $=60 \mathrm{mg} /$ day $)$ and flucytosine $(4 \times 2.25 \mathrm{~g} /$ day $)$. Repeated testing for HIV produced negative results. An external ventricular drainage was placed to decrease the persistently elevated intracranial pressure (Figure 1). Once a substantial improvement in clinical condition had been achieved, after 2 months, the external ventricular shunt was removed, and drug therapy was changed back to oral fluconazole ( $800 \mathrm{mg} /$ day). The cryptococcal CSF antigen titer had decreased to 1:32, while the serum antigen titer was still 1:1,024. A week later, the patient complained of headaches again and became confused. The cryptococcal antigen titer in the CSF had increased from 1:32 to 1:512, the protein was highly elevated, and glucose was non-detectable. Therapy with amphotericin B iv (60 mg/day) was resumed for 2 weeks and the dose of oral fluconazole was augmented $(2 \times 800 \mathrm{mg} /$ day $)$. A new external shunt was put in place for an additional month. The high dose of fluconazole being administered caused the patient's steroid synthesis to be blocked, and she developed symptoms and signs of hypocorticism (hypotonia, hypoglycaemia, dark staining of the skin). This resolved when a substitution with corticosteroids (hydrocortisone $100 \mathrm{mg} /$ day) was started. At the time of discharge, after 132 days of hospitalization, the patient was taking fluconazole $600 \mathrm{mg} /$ day and corticosteroids (hydrocortisone $75 \mathrm{mg} /$ day). Her only manifestation was a slight tremor and she had no further neurological deficits; the cryptococcal antigen titer in the serum was 1:256. The shunt had been removed 5 weeks prior to discharge, and no further measurement of CSF pressure had taken place.

At the last visit as an outpatient in May 2008 the patient was doing well without any neurologic impairment or headache. She has restarted working for $3 \mathrm{~h}$ four times a week. The serum antigen titer was 1:8. She is still taking oral fluconazole at a dose of $600 \mathrm{mg} /$ day $(400 \mathrm{mg}$ in the morning, and $200 \mathrm{mg}$ in the evening).

\section{Discussion}

Most C. gattii infections in humans have been reported from tropical and subtropical areas, mainly Australia and the South Pacific but also Cambodia, Latin America, the USA, and Sub-Saharian Africa $[4,5]$. Only a few infections have been reported from countries with a moderatetemperate climate. In 1999, a C. gattii outbreak emerged on Vancouver Island, Canada [3]. Although rare autochthonous infections due to $C$. gattii in Europe have been reported [2, 10-12], most infections in European residents were acquired outside Europe. The sequence of both the ITS regions and the IGS1 region of the cryptococcal isolate obtained from our patient was found to be identical with those of the genotype VGII isolates which caused the Vancouver Island outbreak [8, 9, 13]. Thus, it is likely that she acquired her infection while visiting Vancouver 
Island in August 2005, 13 months prior to the onset of symptoms. Earlier cases of $C$. gattii infection in travelers to Vancouver Island have shown that the incubation periods of $C$. gattii infection range from 2 to 11 months [14]. This case demonstrates that a careful travel history in which the itineraries of past holidays and journeys to non-tropical regions should be included in the medical history.

The cryptococcal antigen test for sera and CSF is an easy-to-perform and highly sensitive diagnostic test for cryptococcosis. However, the clinician has to be aware of the fact that, in rare instances, immunocompetent patients can also suffer from cryptococcosis and therefore request the test when this infection is suspected. The antigen test is not able to identify the etiologic agent at the species level. The correct species assignment of cryptococcal isolates in apparently immunocompetent patients, however, is important, not only for epidemiological reasons, but also for prognosis and eventual treatment duration. C. gattii infections tend to follow a more chronic course in immunocompetent people than infections with $C$. neoformans [4], and a slower response and worse prognosis have been noted with the former $[4,15]$. Identification in countries with a low prevalence of $C$. gattii mainly relies on biochemical methods, such as the Auxacolor (Sanofi Diagnostics Pasteur) or the ID32C (Biomérieux, Brussels, Belgium) systems. These routine diagnostic procedures, however, do not allow $C$. gattii to be distinguished from $C$. neoformans. Additional efforts are required for assigning an isolate to $C$. gattii, such as growth on CGB medium, D-Prolin assimilation, and IGSand ITS-sequencing.

In terms of antifungal therapy, the combination of amphotericin and flucytosine is accepted as being the most rapid fungicidal therapeutic regimen in the initial 2 weeks of treatment [16]. The goal is to achieve sterilization of the CSF. According to the "practice guidelines for the management of cryptococcal disease" [17], immunocompetent hosts with CNS infection should be treated for 6-10 weeks with amphotericin B iv (0.7-1 mg/ $\mathrm{kg}$ daily) and flucytosine iv (100 mg/kg daily) or with amphotericin B and flucytosine iv (same concentrations) followed by oral fluconazole at a dose of $400 \mathrm{mg} / \mathrm{d}$ for a minimum of another 10 weeks. After that, a fluconazole consolidation therapy for another 6-12 months should be considered, but data are lacking. Recommendations are extrapolated from the treatment experience of HIV-positive patients with $C$. neoformans meningitis. In retrospect, the initial treatment of this patient was incorrect, erroneously based on the appearance of the patient, who did not seem to be severely ill at the time of presentation. Species identification was obtained only during the course of care.

Little is known on the management of increased CSF pressure. Current recommendations are based on a few case reports, one small series, and expert opinion [18].
Clinical observations have revealed that patients infected with $C$. gattii have a higher rate of hydrocephalus requiring shunting [18]. The mechanism is hypothesized to be the obstruction of CSF outflow by to a large burden of yeasts and polysaccharide plugging the arachnoid granulations. The clinician can either perform daily serial lumbar punctures or insert a temporary lumbar drain [19, 20]. If patients present with classical obstructive hydrocephalus, neurosurgical shunting can improve neurological outcome [18]. Aggressive management of elevated intracranial pressure, with repeated lumbar punctures or drainage, is one of the most important approaches in reducing mortality and minimizing morbidity in patients with cryptococcal meningitis. Medical approaches for the management of elevated intracranial pressure, such as the use of corticosteroids or acetazolamid, have not been found to be effective.

In conclusion, the differential diagnosis of cryptococcal meningoencephalitis also needs to be considered in immunocompetent hosts, particularly in the case of negative bacterial and virological findings. In such cases, $C$. gattii should be considered as the etiologic agent, and correct species assignment should be attempted with additional phenotypical and molecular techniques, such as IGS-sequencing and ITS-subtyping. The case presented here demonstrates the importance of a carefully taken travel history.

\section{References}

1. Kwon-Chung KJ, Varma A: Do major species concepts support one, two or more species within Cryptococcus neoformans? FEMS Yeast Res 2006; 6: 574-587.

2. Baro T, Torres-Rodriguez JM, De Mendoza MH, Morera Y, Alia C: First identification of autochthonous Cryptococcus neoformans var. gattii isolated from goats with predominantly severe pulmonary disease in Spain. J Clin Microbiol 1998; 36: 458-461.

3. Hoang LM, Maguire JA, Doyle P, Fyfe M, Roscoe DL: Cryptococcus neoformans infections at Vancouver Hospital and Health Sciences Centre (1997-2002): epidemiology, microbiology and histopathology. J Med Microbiol 2004; 53: 935-940.

4. Mitchell DH, Sorrell TC, Allworth AM, Heath CH, McGregor AR, Papanaoum K, Richards MJ, Gottlieb T: Cryptococcal disease of the CNS in immunocompetent hosts: influence of cryptococcal variety on clinical manifestations and outcome. Clin Infect Dis 1995; 20: 611-616.

5. Sugita $T$, Ikeda R, Shinoda T: Diversity among strains of Cryptococcus neoformans var. gattii as revealed by a sequence analysis of multiple genes and a chemotype analysis of capsular polysaccharide. Microbiol Immunol 2001; 45: 757-768.

6. Diaz MR, Boekhout T, Kiesling T, Fell JW: Comparative analysis of the intergenic spacer regions and population structure of the species complex of the pathogenic yeast Cryptococcus neoformans. FEMS Yeast Res 2005; 5: 1129-1140.

7. Lemmer K, Naumann D, Raddatz B, Tintelnot K: Molecular typing of Cryptococcus neoformans by PCR fingerprinting, in comparison with serotyping and Fourier transform infraredspectroscopy-based phenotyping. Med Mycol 2004; 42: 135-147. 
8. Katsu M, Kidd S, Ando A, Moretti-Branchini ML, Mikami Y, Nishimura K, Meyer W: The internal transcribed spacers and $5.8 \mathrm{~S}$ rRNA gene show extensive diversity among isolates of the Cryptococcus neoformans species complex. FEMS Yeast Res 2004; 4: 377-388.

9. Bovers $M$, Hagen F, Kuramae EE, Boekhout T: Six monophyletic lineages identified within Cryptococcus neoformans and Cryptococcus gattii by multi-locus sequence typing. Fungal Genet Biol 2008; 45: 400-421.

10. Dromer F, Mathoulin S, Dupont B, Laporte A: Epidemiology of cryptococcosis in France: a 9-year survey (1985-1993). French Cryptococcosis Study Group. Clin Infect Dis 1996; 23: 82-90.

11. Kohl KH, Hof H, Schrettenbrunner A, Seeliger HP, Kwon-Chung $\mathrm{KJ}$ : Cryptococcus neoformans var. gattii in Europe. Lancet 1985; 1: 1515 .

12. Tintelnot K, Lemmer K, Losert H, Schar G, Polak A: Follow-up of epidemiological data of cryptococcosis in Austria, Germany and Switzerland with special focus on the characterization of clinical isolates. Mycoses 2004; 47: 455-464.

13. Kidd SE, Hagen F, Tscharke RL, Huynh M, Bartlett KH, Fyfe M, Macdougall L, Boekhout T, Kwon-Chung KJ, Meyer W: A rare genotype of Cryptococcus gattii caused the cryptococcosis outbreak on Vancouver Island (British Columbia, Canada). Proc Natl Acad Sci USA 2004; 101: 17258-17263.
14. MacDougall L, Fyfe M: Emergence of Cryptococcus gattii in a novel environment provides clues to its incubation period. J Clin Microbiol 2006; 44: 1851-1852.

15. Lui G, Lee N, Ip M, Choi KW, Tso YK, Lam E, Chau S, Lai R, Cockram CS: Cryptococcosis in apparently immunocompetent patients. O J Med 2006; 99: 143-151.

16. Brouwer AE, Rajanuwong A, Chierakul W, Griffin GE, Larsen RA, White NJ, Harrison TS: Combination antifungal therapies for HIV-associated cryptococcal meningitis: a randomised trial. Lancet 2004; 363: 1764-1767.

17. Saag MS, Graybill RJ, Larsen RA, Pappas PG, Perfect JR, Powderly WG, Sobel JD, Dismukes WE: Practice guidelines for the management of cryptococcal disease. Infectious Diseases Society of America. Clin Infect Dis 2000; 30: 710-718.

18. Bicanic T, Harrison TS: Cryptococcal meningitis. Br Med Bull 2004; 72: 99-118.

19. Fessler RD, Sobel J, Guyot L, Crane L, Vazquez J, Szuba MJ, Diaz FG: Management of elevated intracranial pressure in patients with Cryptococcal meningitis. J Acquir Immune Defic Syndr Hum Retrovirol 1998; 17: 137-142.

20. Macsween KF, Bicanic T, Brouwer AE, Marsh H, Macallan DC, Harrison TS: Lumbar drainage for control of raised cerebrospinal fluid pressure in cryptococcal meningitis: case report and review. J Infect 2005; 51: 221-224. 